\title{
Actinomycosis of the Brain and Temporal Bone in a Goat
}

\author{
Takuya HIRAI ${ }^{1)}$, Tetsuo NUNOYA ${ }^{1)}$ and Ryozo AZUMA ${ }^{2)}$ \\ ${ }^{1)}$ Nippon Institute for Biological Science, 9-2221-1 Shinmachi, Ome, Tokyo 198-0024 and 2) Junior College of Tokyo University of \\ Agriculture, 1-1-1 Sakuragaoka, Setagayaku, Tokyo 156-0054, Japan
}

(Received 22 November 2006/Accepted 29 January 2007)

\begin{abstract}
A goat with neurologic signs had multifocal abscesses containing sulfur granules in the right brain and temporal bone. Histologically, the lesions consisted of pyogranulomas with several radiating bacterial colonies of various sizes. A tangled mass of filamentous and gram-positive bacteria was recognized in the central part of the colony. Actinomyces naeslundii antigen was detected in the colonies of bacteria in the brain and neighboring bone tissue by immunohistochemistry. Actinomycosis involving the central nervous system (CNS) and temporal bone is rare in animals. Cerebral infection with $A$. naeslundii may have resulted from direct extension from cervicofacial regions because the CNS lesions were distributed asymmetrically and were continuous with the right temporal bone. KEY WORDS: actinomycosis, brain, goat.
\end{abstract}

Actinomycosis is a chronic suppurative infection caused by an anaerobic, gram-positive, non acid-fast, filamentous bacterium [2, 3, 9, 10]. Although Actinomyces is part of the normal flora of oral cavity, it has been known to cause endogenous infections, usually after minor trauma resulting in mucosal breaks $[3,9,10]$. Actinomyces bovis is the most common pathogen found in cattle $[3,8]$. The disease, commonly called "lumpy jaw", is a mandibular pyogranulomatous osteomyelitis $[3,8]$. In both animals and humans, central nervous system (CNS) infection with Actinomyces is rare and usually results from extension of an adjacent focus or from hematogenous seeding from a distant site $[1,2,6$, 9]. Predisposing risk factors include dental caries or infections, tooth extraction, gastrointestinal tract surgery, chronic otitis, mastoiditis or sinusitis, chronic osteomyelitis, tetralogy of Fallot, and Actinomyces infection of an intrauterine device [9]. According to microbiologic data for patients with CNS actinomycosis, $A$. israelii was the most commonly identified causative organism [9]. This report describes a case of pyogranulomatous meningoencephalitis and osteomyelitis of the temporal bone due to A. naeslundii infection in a goat.

A 6-year-old goat was submitted to our institute for pathological diagnosis because of general ataxia. At necropsy, the superficial blood vessels of the cerebral cortex were hyperemic. A $5.5 \times 2.5 \times 1.0 \mathrm{~cm}$ mass was located in the right cerebral hemisphere and right temporal bone. Sagittal sections showed multiple abscesses containing yellowish colored sulfur granules in both the right cerebrum and the mass (Fig. 1). Except for the brain and the skull bone, no other abnormalities were found in other organs and tissues.

Tissue samples were fixed in $10 \%$ phosphate-buffered formalin, embedded in paraffin wax, sectioned at $5 \mathrm{~mm}$, and stained with hematoxylin and eosin (HE) for light microscopy. Selected brain sections were also stained with Gram's (MacCallum-Goodpasture), Grocott's and Ziehl-Neelsen methods. Paraffin wax-embedded brain tissues were immunohistochemically examined with the streptavidin-biotin method (Nichirei, Inc., Japan). Sera from rabbits hyperimmunized with $A$. naeslundii, A. bovis, $A$. viscosus and $A$. suis were used as primary antibodies $[4,5]$.

Histopathologically, multifocal pyogranulomas were present in the right cerebrum, meninges and temporal bone. Bone lysis and irregular new bone formation were observed in the affected temporal bone. The central abscesses were surrounded by epithelioid macrophages, lymphocytes and occasional giant cells of the Langhans' type. A distinctive morphologic feature of the pyogranulomas was the presence of irregularly shaped eosinophilic colonies surrounded by radially arranged projections known as Splendore-Hoeppli material (Fig. 2). The microorganisms in the lesions were positively stained by Gram's and Grocott's stains (Fig. 3) but negatively by Ziehl-Neelsen method. The bacteria were filamentous, peripherally beaded and branched. These findings were consistent with those of Actinomyces sp. The microorganisms in the lesions were intensely immun-

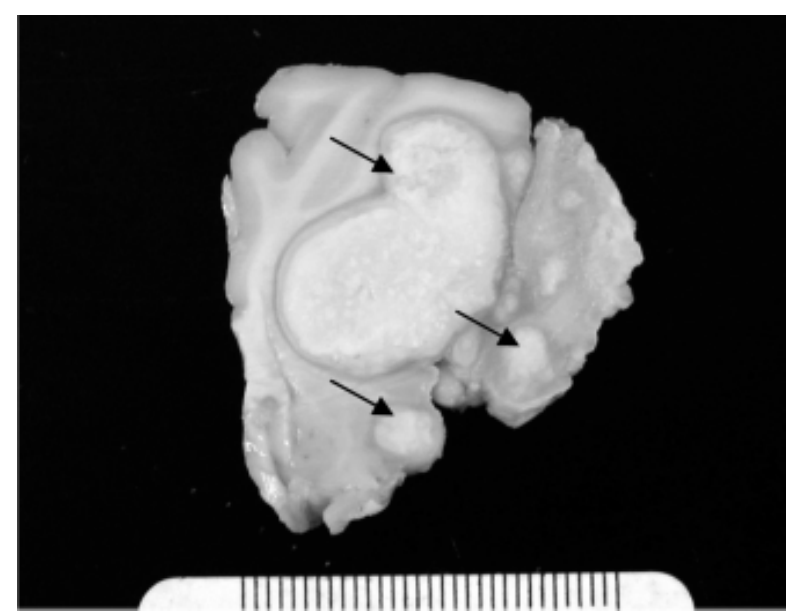

Fig. 1. Transverse section of the frontal lobe of the right brain and neighbouring bone tissues. Note multifocal abscesses (arrows) containing yellowish colored sulfur granules. 


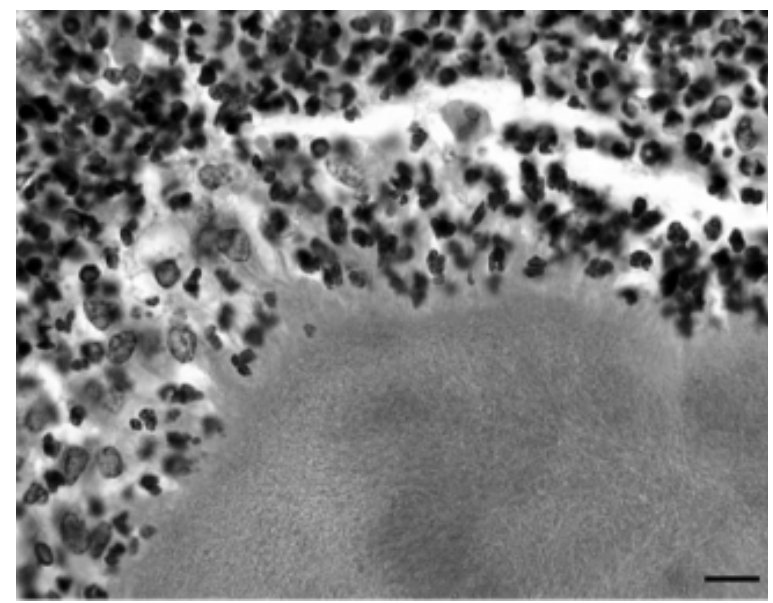

Fig. 2. Section of the brain showing radiating bacterial colony in the pyogranuloma. Splendore-Hoeppli material around the bacterial colonies. Hematoxylin and Eosin stain. Bar, $10 \mu \mathrm{m}$.

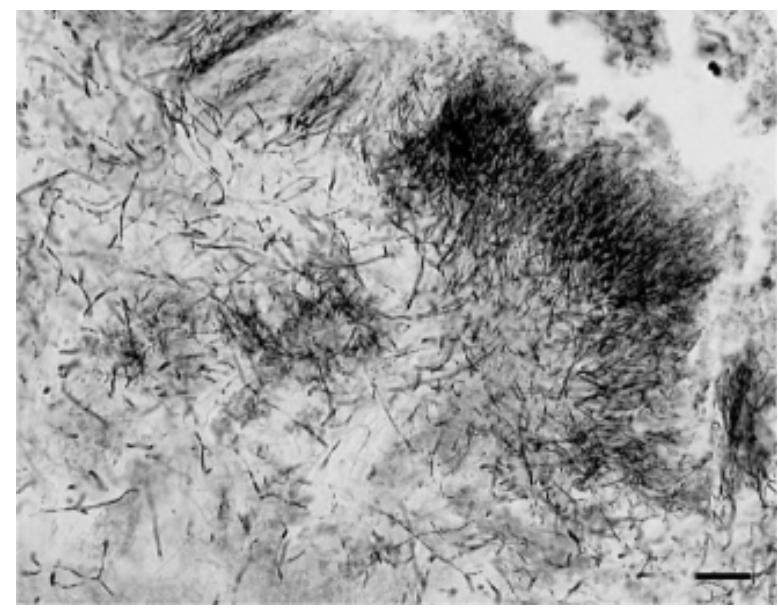

Fig. 3. Note tangled or branching filamentous microorganisms in the pyogranuloma. Grocott's stain. Bar, $10 \mu \mathrm{m}$.

ostained for A. naeslundii (Fig. 4), and were negative when the primary antiserum was substituted with phosphatebuffer saline. No immunolabelling of the microorganisms was found for A. bovis, A. viscosus and A. suis.

Our differential diagnosis included nocardiosis, which produces lesions similar to those of actinomycosis and often causes lesions of the CNS in both animals and man $[6,9]$. Nocardia are aerobic, gram-positive and filamentous organisms that have 'acid fast" staining properties [3]. The filamentous microorganisms in the pyogranulomas were not stained by the Ziehl-Neelsen method in the present case. These results support our diagnosis of an A. naeslundii infection in a goat.

In humans, A. naeslundii inhabits the oral cavity including tonsillar crypts and dental plaque and is also detected in cervicovaginal secretions from women [7]. On the other hand, A. naeslundii has been identified as the causative

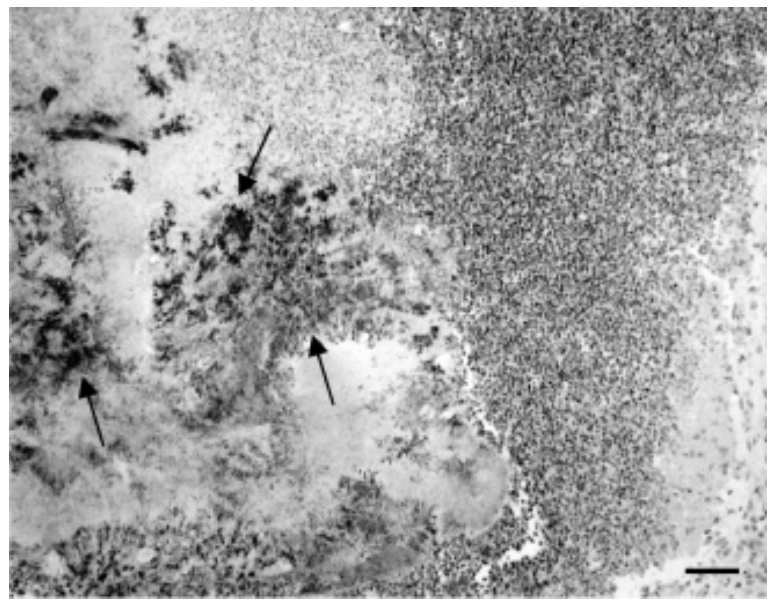

Fig. 4. Immunohistochemical detection of Actinomyces naeslundii antigens (arrows) in the pyogranuloma of brain. Hematoxylin counterstain. Immunohistochemistry. Bar, $50 \mu \mathrm{m}$.

agent of actinomycosis in humans and animals $[3,4,7]$. The most common sites of $A$. naeslundii infection in human are the cervicofacial region, the abdomen and the thorax [7]. Furthermore, A. naeslundii obviously plays an important role in the complex etiology and pathogenesis of caries and periodontal disease [7]. In swine, A. naeslundii has been associated with abortion, by causing typical actinomycotic lesions in the uterus and the fetal lungs [3]. In the present case, it is likely that $A$. naeslundii comprises a part of the normal flora in the oral cavity of goats and gains entrance for infection from damaged tissue of the oral cavity. The cerebral infection with $A$. naeslundii might have resulted from direct extension from the cervicofacial regions because CNS lesions were distributed asymmetrically and were continuous with the right temporal bone. Fewer than 30 cases of actinomycosis involving the temporal bone have been reported in humans [10]. In those cases, the infection is believed to be occurred by invasion of the organism from the pharynx to the middle ear via the eustachian tube [10]. The same infectious route may have been used in the present case. Unfortunately, the precise primary site of infection was not defined in the present case because the mandible, tonsils, pharynx, middle ears and paranasal sinuses were not examined carefully.

Several cases of Actinomyces causing CNS disease have been reported in animals: spinal meningitis in an Arctic fox, encephalitis associated with hydrocephalus in a dog and pyogranulomatous meningoencephalitis in a $\operatorname{dog}[1,2,6]$. The former two cases had facial or head bite wounds that probably were the source of infection, but identification of the organisms could not be made in all these cases $[1,2,6]$. This report presents a rare case of CNS and temporal bone actinomycosis caused by $A$. naeslundii in a goat.

ACKNOWLEDGMENT. The authors would like to thank Ms Hitomi Tomioka for her excellent technical assistance. 


\section{REFERENCES}

1. Anvik, J. O. and Lewis, R. 1976. Can. Vet. J. 17: 42-44.

2. Couto, S. S., Dickinson, P. J., Jang, S. and Munson, L. 2000. Vet. Pathol. 37: 650-652.

3. Jones, T. C., Hunt, R. D. and King, N. W. 1997. pp. 482-486. In: Veterinary Pathology, 6th ed., Williams \& Wilkins, Baltimore.

4. Kawamura, N., Shimada, A., Morita, T., Murakami, S. Azuma, R., Fujiwara, M. and Fujiwara, A. 2005. Vet. Rec. 157: 593-594.

5. Murakami, S., Yamanishi, M. W. and Azuma, R. 1997. J. Vet.
Med. Sci. 59: 1079-1080.

6. Raju, N. R., Langham, R. F., Kispert, C. and Koestner, A. 1986. J. Am. Vet. Med. Assoc. 189: 1194-1195.

7. Schaal, K. P. 1986. pp. 1383-1417. In: Bergey's Manual of Systemic Bacteriology. 1st ed., Williams \& Wilkins, Baltimore.

8. Seifi, H. A., Saifzadeh, S., Farshid, A. A., Rad, M. and Farrokhi, F. 2003. J. Vet. Med. A 50: 219-221.

9. Smego, R. A. Jr. 1987. Rev. Infect. Dis. 9: 855-865.

10. Sobol, S. E., Samadi, D. S. and Wetmore, R. F. 2004. Ear. Nose. Throat. J. 83: 327-329. 